\title{
Phase II study of capecitabine and oxaliplatin given prior to and concurrently with preoperative pelvic radiotherapy in patients with locally advanced rectal cancer
}

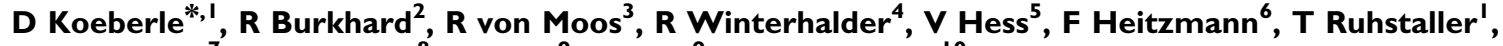

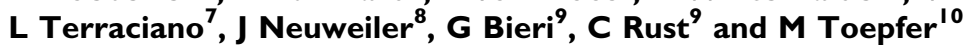

'Division Oncology/Hematology, Department of Internal Medicine, Kantonsspital St Gallen, St Gallen CH-9007, Switzerland; ${ }^{2}$ Department of Internal Medicine, Stadtspital Triemli, Zürich CH-8063, Switzerland; ${ }^{3}$ Department of Medical Oncology, Kantonsspital Graubünden, Chur CH-7000, Switzerland; ${ }^{4}$ Department of Internal Medicine, Kantonsspital Luzern, Luzern CH-6000, Switzerland; ${ }^{5}$ Department of Medical Oncology, Universitätsspital Basel, Basel CH-403I, Switzerland; ' ${ }^{6}$ Stadtspital Waid, Department of Internal Medicine, Zurich CH-8037, Switzerland; ${ }^{7}$ Universitätsspital Basel, Department of Clinical Pathology, Basel CH-403 I, Switzerland; ${ }^{8}$ Kantonsspital St Gallen, Department of Pathology, St Gallen CH-9007, Switzerland; ${ }^{9}$ Roche Pharma (Schweiz) AG, Reinach CH-4 153, Switzerland; ${ }^{10}$ Department of Radio-Oncology, Kantonsspital St Gallen, St Gallen CH-9007, Switzerland

This multicentre phase II study evaluated the efficacy and safety of preoperative capecitabine plus oxaliplatin and radiotherapy (RT) in patients with locally advanced rectal cancer (T3/T4 rectal adenocarcinoma with or without nodal involvement). Treatment consisted of one cycle of XELOX (capecitabine $1000 \mathrm{mg} \mathrm{m}^{-2}$ bid on days I- 14 and oxaliplatin $130 \mathrm{mg} \mathrm{m}^{-2}$ on day I), followed by RT (I.8 Gy fractions 5 days per week for 5 weeks) plus CAPOX (capecitabine $825 \mathrm{mg} \mathrm{m}^{-2}$ bid on days $22-35$ and $43-56$, and oxaliplatin $50 \mathrm{mg} \mathrm{m}^{-2}$ on days 22, 29, 43 and 50). Surgery was recommended 5 weeks after completion of chemoradiotherapy. The primary end point was pathological complete tumour response ( $\mathrm{PCR}$ ). Sixty patients were enrolled. In the intent-to-treat population, the pCR rate was 23\% (95\% Cl: 13-36\%). 58 patients underwent surgery; R0 resection was achieved in 57 (98\%) patients, including all 5 patients with T4 tumours. Sphincter preservation was achieved in 49 (84\%) patients. Tumour and/or nodal downstaging was observed in 39 (65\%) patients. The most common grade 3/4 adverse events were diarrhoea (20\%) and lymphocytopaenia (43\%). Preoperative capecitabine, oxaliplatin and RT achieved encouraging rates of $\mathrm{pCR}$, RO resection, sphincter preservation and tumour downstaging in patients with locally advanced rectal cancer.

British Journal of Cancer (2008) 98, I204- 1209. doi:I0.1038/sj.bjc.6604297 www.bjcancer.com

Published online 18 March 2008

(c) 2008 Cancer Research UK

Keywords: rectal cancer; radiochemotherapy; capecitabine; oxaliplatin

In recent years, considerable progress has been made in the treatment of locally advanced rectal cancer, mainly due to improvements in the type and quality of surgery, better staging methods and regular use of chemoradiation (CRT) or radiation therapies. Although the use of preoperative CRT for resectable rectal cancer remains a controversial issue, preoperative CRT is clearly preferred when tumour shrinkage is required before surgery, that is, in locally advanced T4 disease and low-lying tumours when sphincter preservation is attempted (Sauer et al, 2004; Bosset et al, 2006; Gérard et al, 2006). Furthermore, preoperative CRT improves local disease control with less toxicity compared with postoperative CRT (Sauer et al, 2004).

Many attempts have been made to increase the convenience and activity of preoperative 5-fluorouracil (5-FU)-based CRT. Evidence from phase II trials suggests that the oral fluoropyrimidine

*Correspondence: Dr D Koeberle; E-mail: dieter.koeberle@kssg.ch Received 15 November 2007; revised 14 February 2008; accepted 21 February 2008; published online 18 March 2008 capecitabine (Xeloda ${ }^{\circledR}$; F Hoffmann-La Roche Ltd, Basel, Switzerland) has similar activity to that of protracted 5-FU infusional CRT regimens (Glynne-Jones et al, 2006a). Combining different chemotherapy agents, such as oxaliplatin or irinotecan, with fluoropyrimidines has a clear rationale based on a plethora of data in the metastatic colorectal setting and the potential to further improve efficacy in patients receiving preoperative CRT. Oxaliplatin (Eloxatin ${ }^{\circledR}$; Sanofi-Aventis, Bridgewater, NJ, USA) is an ideal candidate for inclusion into neoadjuvant CRT regimens because of its radiosensitising capabilities and synergy with fluoropyrimidines.

Capecitabine has been tested in combination with oxaliplatin and radiotherapy in several different regimens (for review see Glynne-Jones et al, 2006a). These include continuous capecitabine (7 days per week) with oxaliplatin given on days 1 and 29 (Glynne-Jones et al, 2006c), continuous capecitabine (5 days per week) with weekly doses of oxaliplatin (Machiels et al, 2005; Rutten et al, 2006) and discontinuous capecitabine (days $1-14$ and $22-35$ ) with oxaliplatin on days $1,8,22$ and 29 (Roedel et al, 2003, 2007). The aim of the present multicentre phase II study was to 
Table I Histological tumour regression grading systems

\begin{tabular}{|c|c|}
\hline Grade & Definition \\
\hline \multicolumn{2}{|c|}{ Dworak et al (1997) } \\
\hline 0 & No regression \\
\hline I & Dominant tumour mass with obvious fibrosis and/or vasculopathy \\
\hline 2 & $\begin{array}{l}\text { Dominantly fibrotic changes with few tumour cells or groups } \\
\text { (easy to find) }\end{array}$ \\
\hline 3 & $\begin{array}{l}\text { Very few (difficult-to-find microscopically) tumour cells in fibrotic tissue } \\
\text { with or without mucous substance }\end{array}$ \\
\hline 4 & No tumour cells, only fibrotic mass (total regression or response) \\
\hline \multicolumn{2}{|c|}{ Mandard et al (1994) (adapted by Bouzourene et al, 2002) } \\
\hline 5 & Absence of regressive changes \\
\hline 4 & Residual cancer outgrowing fibrosis \\
\hline 3 & $\begin{array}{l}\text { Increase in the number of residual cancer cells, but fibrosis still } \\
\text { predominant }\end{array}$ \\
\hline 2 & Presence of rare residual cancer cells scattered through the fibrosis \\
\hline 1 & $\begin{array}{l}\text { Complete regression, absence of histologically identifiable residual } \\
\text { cancer and fibrosis extending through the different layers of the rectal } \\
\text { wall, with or without granuloma }\end{array}$ \\
\hline
\end{tabular}

carcinomas, which was subsequently adapted by Bouzourene et al (2002) (see Table 1 for summary).

At each site, one preselected pathologist evaluated the tumour tissue of all patients participating in the study. A second-opinion pathology review was performed in all tumours categorised as Dworak grade 2 or 3 by a pathologist from another centre participating in the study.

Safety was assessed through documenting adverse events and clinical laboratory tests performed at screening, during treatment and the surgery period. Adverse events were graded using NCI CTC version 3.0

\section{Statistical analysis}

The intent-to-treat population (ITT) consisted of all patients who received at least one dose of study medication whether or not they were eligible. All efficacy analyses were performed on this population. Patients not undergoing surgery or who were not evaluable for response were considered nonresponders. The safety population consisted of patients who received at least one dose of any study drug and who had a baseline assessment and at least one safety follow-up.

The primary end point was pathological complete tumour response (pCR) prospectively defined as grade 3 or 4 according to the Dworak classification (DC) system (Dworak et al, 1997). The pCR rate was presented with $95 \%$ confidence intervals (CIs) using the Pearson-Clopper method. Secondary end points were rate of sphincter preservation, $\mathrm{R} 0$ resection in patients with $\mathrm{T} 4$ tumours, downstaging (defined as a decrease of $\geqslant 1$ point(s) in $\mathrm{T}$ and/or $\mathrm{N}$ value) and safety.

An exploratory analysis using the Fisher exact test was performed to test for an association between the presence and absence of a complete tumour response (DC grade $3 / 4$ vs $0 / 1 / 2$ ), and site, location and size of primary tumour at screening, $\mathrm{u} /$ cTNM classification at screening, time between radiotherapy and surgery $(\leqslant 40$ vs $>40$ days), age $(\leqslant 60$ vs $>60$ years $)$ and treatment-related lymphocytopaenia.

This study was designed as a one-stage phase II trial using pCR rate as the primary efficacy criterion. A pCR of $22 \%$ was considered acceptable and a rate of $\leqslant 7 \%$ was ruled out as futile. With a total of 48 evaluable patients (and a response rate of at least $22 \%$ ), a power of $86 \%$ and a type-I error of $4.8 \%$ was achieved. The
Table 2 Patient characteristics $(N=60)$

\begin{tabular}{|c|c|c|}
\hline Characteristic & No. of patients & $\%$ \\
\hline \multicolumn{3}{|l|}{ Sex } \\
\hline Male & 46 & 77 \\
\hline Female & 14 & 23 \\
\hline \multicolumn{3}{|l|}{ Age, years } \\
\hline Median (range) & $61(35-76)$ & \\
\hline \multicolumn{3}{|l|}{ u/cTNM classification } \\
\hline T stage 2 & । & 2 \\
\hline T stage 3 & 53 & 88 \\
\hline T stage 4 & 6 & 10 \\
\hline $\mathrm{N}$ stage 0 & 13 & 22 \\
\hline N stage | & 44 & 73 \\
\hline $\mathrm{N}$ stage 2 & 3 & 5 \\
\hline \multicolumn{3}{|l|}{ Location of tumour ${ }^{a}$} \\
\hline Lower rectum $(0-5 \mathrm{~cm})$ & 22 & 37 \\
\hline Middle rectum $(5.1-10 \mathrm{~cm})$ & 30 & 50 \\
\hline Upper rectum $(10.1-12 \mathrm{~cm})$ & 17 & 28 \\
\hline \multicolumn{3}{|l|}{ Size of primary tumour (mm) } \\
\hline Median (range) & $50(16-140)$ & \\
\hline Infiltration of sphincter muscle & 6 & 10 \\
\hline \multicolumn{3}{|l|}{ Intended type of surgery } \\
\hline Total mesorectal excision & 46 & 77 \\
\hline Abdominoperineal resection & 14 & 23 \\
\hline
\end{tabular}

aMore than one location per patient possible.

planned sample size was increased to 60 patients to allow for dropouts.

\section{RESULTS}

\section{Patient characteristics}

A total of 60 patients were enrolled between March 2005 and July 2006 from six cancer centres in Switzerland. Patient characteristics are summarised in Table 2. All 60 patients were included in the safety and ITT populations, including 2 patients who were ineligible (one patient because of cT2 rectal cancer, and the other patient because of an urothelial cancer 4 years before the start of the study). Fifty-eight patients ( $97 \%$ of all recruited patients) received CRT and underwent surgery; one patient withdrew consent and one patient died prior to surgery.

\section{Dose intensity and safety}

Fifty-five patients (92\%) received all three cycles of capecitabine (mean relative dose intensity $97 \%$ ), and 52 patients (87\%) received all five planned oxaliplatin doses (mean relative dose intensity $97 \%$ ). The mean relative dose intensity for capecitabine was $98 \%$ during XELOX and 96\% during CAPOX-RT. For oxaliplatin, the mean relative dose intensity was $99 \%$ during XELOX and $93 \%$ during CAPOX-RT. Fifty-six patients (93\%) received at least 25 fractions $(45 \mathrm{~Gy})$ of radiotherapy as planned.

Table 3 summarises grade $3 / 4$ treatment-related nonhaematological toxicities presented per treatment regimen (XELOX vs CAPOX-RT). The most frequently occurring grade $3 / 4$ adverse event was diarrhoea (20\%); all other grade $3 / 4$ events were uncommon $(\leqslant 5 \%)$. No grade $3 / 4$ haematological toxicity was observed, except for lymphocytopaenia (43\%). At least one serious 
Table 3 Most frequently reported nonhaematological treatment-related adverse events $(N=60)$

Toxicity according to NCI CTC grade (\% of patients)

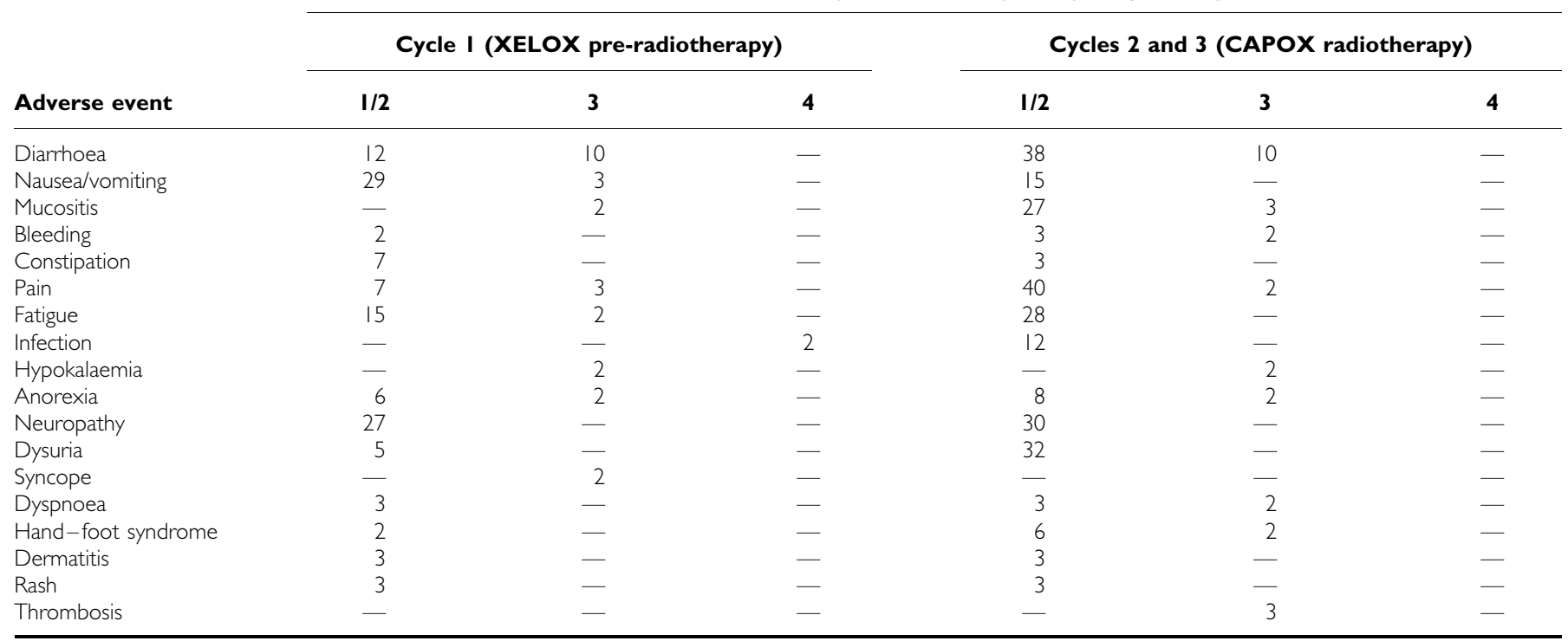

$\mathrm{NCl} \mathrm{CTC}=$ National Cancer Institute Common Terminology Criteria.

adverse event was recorded in eight patients (13\%) during the study. A total of 12 serious adverse events $(20 \%)$ were reported, the most common of which were diarrhoea $(n=5)$ and colitis or proctitis $(n=2)$. One patient developed severe neutropaenic infection and died on day 19 after the start of neoadjuvant XELOX. Four patients $(7 \%)$ had one adverse event leading to discontinuation of capecitabine, and three patients $(5 \%)$ had adverse events leading to discontinuation of oxaliplatin. No patient required discontinuation of radiotherapy.

\section{Efficacy and surgical parameters}

Surgery was performed in a total of 58 patients (TME in 47 patients $(81 \%)$, abdominoperineal extirpation in 9 patients $(16 \%)$ and other type of surgery in 2 patients $(3 \%))$. The median time between the end of radiotherapy and surgery was 42 days (range: $24-59$ days). In 57 patients (98\%), including all 5 patients with c/uT4 tumours, $\mathrm{R} 0$ resection was achieved and sphincter preservation was achieved in 49 patients $(84 \%)$.

Comparing the baseline tumour stage with the pathological stage in the ITT population, downstaging with respect to tumour stage was observed in $28(47 \%)$ patients, and downstaging with respect to nodal stage was observed in $29(48 \%)$ patients. A detailed analysis is shown in Table 4.

In the ITT population, complete tumour regression (ypT0 N0, DC regression grade 4) was achieved in seven patients. An additional seven patients showed near-complete regression (DC regression grade 3 ) with only very few detectable tumour cells as assessed by two independent pathologists. According to predefined criteria, the pCR rate was therefore $23 \%$ (95\% CI: $13-36 \%$ ). The corresponding pCR rate according to the Mandard regression grading system (grades 1 and 2) was 27\% (grade 1, seven patients; grade 2, nine patients).

A second-opinion review of all specimens rated as DC grade 2 or 3 was necessary in 33 cases (57\%). After the second opinion, the final DC grading remained the same in 27 cases (82\%), downgrading was deemed necessary in 5 cases $(15 \%)$ and upgrading in 1 case $(3 \%)$. Both tumour regression scales were compared using the final DC grades and Mandard grades. The scales seemed to correspond well; all patients with DC grade 0 reported Mandard-tumour regression (M-TR) grade 5, 90\% of patients
Table 4 Preoperative T/N stage compared with pathological T/N stage $(N=58)$

\begin{tabular}{lcccccccccc}
\hline $\begin{array}{l}\text { Baseline } \\
\text { staging }\end{array}$ & pT0 & pTis & pTI & pT2 & pT3 & pT4 & pN0 & pNI & pN2 & pNx \\
\hline uT2 & - & - & । & - & - & - & - & - & - & - \\
uT3 & 5 & । & । & 15 & 28 & 2 & - & - & - & - \\
uT4 & I & - & - & 3 & 1 & - & - & - & - & - \\
uN0 & - & - & - & - & - & - & 7 & 3 & 2 & । \\
uNI & - & - & - & - & - & - & 28 & 6 & 7 & । \\
uN2 & - & - & - & - & - & - & $\mid$ & - & 2 & - \\
\hline
\end{tabular}

with DC grade 1 reported M-TR grade $4,74 \%$ of patients with DC grade 2 reported M-TR grade 3, $86 \%$ of patients with DC grade 3 reported M-TR grade 2 and all patients with DC grade 4 reported M-TR grade 1.

According to an exploratory subgroup analysis, only upper location of the primary tumour (between 10 and $12 \mathrm{~cm}$ from anal verge) was found to be negatively correlated with pCR $(P=0.0504)$.

\section{DISCUSSION}

Pathological complete tumour response rates between 10 and $30 \%$ have been observed with combined preoperative chemotherapy and radiotherapy protocols. Pathological complete tumour response is a reliable and reproducible surrogate for tumour response and is linked to improved outcome (Roh et al, 2004; Roedel et al, 2005). Although achievement of a pCR is not the primary goal of neoadjuvant therapy, it has become a commonly used end point in many phase II trials aiming to improve the efficacy of rectal cancer treatment. 
In the present trial, we are able to demonstrate a pCR in $23 \%$ of patients, defined as grades 3 and 4 according to the Dworak classification (Dworak et al, 1997) following preoperative therapy with a single cycle of XELOX and two further cycles of CAPOX given with radiotherapy. Recently, several different tumour regression scales (Mandard et al, 1994; Dworak et al, 1997; Bouzourene et al, 2002; Wheeler et al, 2004) have been proposed for the measurement of regression after preoperative therapies independent of the ypTNM stage. Besides several differences in categorisation of tumour regression, all of the scales acknowledge a distinctive group of tumours with only microscopic foci of remaining tumour cells. We have grouped patients with sterilised primary tumours and lymph nodes (DC grade 4) together with DC grade 3 tumours. This is based on the observation that single residual tumour cells confer a significantly lower local relapse rate and a better prognosis (Wheeler et al, 2004) than tumours with remaining dominant disease.

The second-opinion review was used for all specimens rated to be either DC grade 2 or 3 by the first pathologist. A high concordance rate between independent pathologists of $82 \%$ suggests a reasonable capability to discriminate between very few and difficult-to-find tumour cells (DC grade 3 ) from easy-to-find few tumour cells or groups of tumour cells (DC grade 2). In addition, applying the regression grading classification of Mandard et al (1994) adapted for rectal cancer (Bouzourene et al, 2002) and combining Mandard's grade 1 (absence of residual tumour cells) and grade 2 (rare residual tumour cells), complete tumour response was achieved in $27 \%$ of patients. These almost identical results from two different scoring systems indicate a high reproducibility in defining near-complete and complete sterilisation of tumour cells.

Furthermore, and an important point with regard to prognosis, we observed nodal downstaging in $48 \%$ of patients. The number of tumour-infiltrated lymph nodes (ypN status) following preoperative radiochemotherapy is a strong and independent prognostic factor for survival. Sterilising lymph nodes reflects the impact of effective neoadjuvant treatment and consistently translates into improved long-term outcome (Bouzourene et al, 2002; Chan et al, 2005; Chapet et al, 2005; Roedel et al, 2005).

In $98 \%$ of the 58 patients who underwent surgery and in all patients with T4 rectal cancer, R0 resection was possible. Sphincter preservation was achieved in $84 \%$ of patients. This appears remarkable as $35 \%$ of our patients had low-lying tumours $(0-5 \mathrm{~cm}$ from anal verge).

The most common nonhaematological toxicity in our trial was grade 3 or 4 diarrhoea, which occurred overall in $20 \%$ of patients (10\% during XELOX and 10\% during CAPOX-RT). This rate is slightly higher than that reported by Roedel et al $(2003,2007)$ and suggests that the additional cycle of XELOX increased the toxicity of preoperative CRT. All other toxicities were in the range of other trials with the exception of grade 3 or 4 lymphocytopaenia.
Lymphocytopaenia is a negative prognostic factor in cancer patients and can be induced by either chemotherapy or pelvic radiotherapy. Decline in total lymphocyte counts is obviously an underreported toxicity and seems to be negatively correlated with tumour regression following pelvic radiotherapy (Lissoni et al, 2005).

The addition of a single chemotherapy cycle before CRT does not appear to have substantially enhanced the overall antitumour activity and should, therefore, not be considered as an important treatment element. In our trial, we added this chemotherapy cycle primarily to assure early start of therapy. Even though many patients reported improvement in symptoms before starting CRT (data not shown), we did not consider achieving a relevant downsizing effect with a single chemotherapy cycle.

The use of neoadjuvant chemotherapy prior to preoperative CRT in rectal cancer patients is a matter of debate (Glynne-Jones and Sebag-Montefiore, 2006; Glynne-Jones et al, 2006b) primarily because satisfactory local control rates can be achieved with preoperative CRT alone. Chau et al (2006) questioned this position by adding four cycles of neoadjuvant capecitabine and oxaliplatin before CRT with capecitabine in their trial. Most patients $(86 \%)$ had symptomatic responses, and the radiological response rate measured by MRI was $88 \%$. Pathological complete tumour response was achieved in $24 \%$ of patients, which is clearly superior to the $11 \%$ DC regression grade 4 in our trial. However, 4 out of 77 patients died during neoadjuvant chemotherapy. In the absence of a randomised phase III trial proving superior outcome, the addition of primary chemotherapy to CRT should be only used in the context of clinical trials.

A different treatment strategy, in an attempt to increase the quantity of systemic treatment, was studied by Roedel et al (2007). After preoperative capecitabine/oxaliplatin radiotherapy, $60 \%$ of patients received all four cycles of adjuvant capecitabine/ oxaliplatin underlining the feasibility of delivering adequate doses of postoperative combination chemotherapy in rectal cancer patients.

In conclusion, we demonstrated that preoperative XELOX followed by CAPOX-RT is feasible with manageable toxicity and results in encouragingly high rates of $\mathrm{pCR}, \mathrm{R} 0$ resection, sphincter preservation and tumour downstaging in patients with locally advanced rectal cancer. More importantly, we were able to replicate, and thus confirm the findings from Roedel et al (2003, 2007) in a multicentre setting in Switzerland.

\section{ACKNOWLEDGEMENTS}

We greatly appreciate the help of Mr Lee Miller in preparing this manuscript. The study was sponsored by Roche Pharma (Schweiz) AG and Sanofi-Aventis (Schweiz) AG.

\section{REFERENCES}

Bosset JF, Collette L, Calais G, Mineur L, Maingon P, Radosevic-Jelic L, Daban A, Bardet E, Beny A, Ollier JC (2006) Chemotherapy with preoperative radiotherapy in rectal cancer. $N$ Engl J Med 355: $1114-1123$

Bouzourene H, Bosman FT, Seelentag W, Matter M, Coucke P (2002) Importance of tumor regression assessment in predicting the outcome of patients with locally advanced rectal carcinoma who are treated with preoperative radiotherapy. Cancer 94: $1121-1130$

Cassidy J, Tabernero J, Twelves C, Brunet R, Butts C, Conroy T, Debraud F, Figer A, Grossmann J, Sawada N, Schöffski P, Sobrero A, Van Cutsem E, Díaz-Rubio E (2004) XELOX (capecitabine plus oxaliplatin): active first-line therapy for patients with metastatic colorectal cancer. J Clin Oncol 22: 2084-2091

Chan AK, Wong A, Jenken D, Heine J, Buie D, Johnson D (2005) Posttreatment TNM staging is a prognostic indicator of survival and recurrence in tethered or fixed rectal carcinoma after preoperative chemotherapy and radiotherapy. Int J Radiat Oncol Biol Phys 61: $665-677$

Chapet O, Romestaing P, Mornex F, Souquet JC, Favrel V, Ardiet JM, d'Hombres A, Gerard JP (2005) Preoperative radiotherapy for rectal adenocarcinoma: which are strong prognostic factors? Int J Radiat Oncol Biol Phys 61: 1371-1377 
Chau I, Brown G, Cunningham D, Tait D, Wotherspoon A, Norman AR, Tebbutt N, Hill M, Ross PJ, Massey A, Oates J (2006) Neoadjuvant capecitabine and oxaliplatin followed by synchronous chemoradiation and total mesorectal excision in magnetic resonance imaging-defined poor-risk rectal cancer. J Clin Oncol 24: 668-674

Díaz-Rubio E, Evans TR, Tabernero J, Cassidy J, Sastre J, Eatock M, Bisset D, Regueiro P, Baselga J (2002) Capecitabine (Xeloda) in combination with oxaliplatin: a phase I, dose-escalation study in patients with advanced or metastatic solid tumors. Ann Oncol 13: 558-565

Dworak O, Keilholz L, Hoffmann A (1997) Pathological feature of rectal cancer after preoperative radiochemotherapy. Int $J$ Colorectal Dis 12: $19-23$

Gérard TC, Conroy T, Bonnetain F, Bouché O, Chapet O, Closon-Dejardin MT, Untereiner M, Leduc B, Francois E, Maurel J, Seitz JF, Buecher B, Mackiewicz R, Ducreux M, Bedenne L (2006) Preoperative radiotherapy with or without concurrent fluorouracil and leucovorin in T3-4 rectal cancers: results of FFCD 9203. J Clin Oncol 24: 4620-4625

Glynne-Jones R, Dunst J, Sebag-Montefiore D (2006a) The integration of oral capecitabine into chemoradiation regimens for locally advanced rectal cancer: how successful have we been? Ann Oncol 17: 361-371

Glynne-Jones R, Grainger J, Harrison M, Ostler P, Makris A (2006b) Neoadjuvant chemotherapy prior to preoperative chemoradiation or radiation in rectal cancer: should we be more cautious? Br J Cancer 94: $363-371$

Glynne-Jones R, Sebag-Montefiore D (2006) Role of neoadjuvant chemotherapy in rectal cancer: interpretation of the EXPERT study. J Clin Oncol 24: 4664-4665

Glynne-Jones R, Sebag-Montefiore D, McDonald A, Maughan TS, Falk SJ, McDonald AC (2006c) A phase I dose escalation study of continuous oral capecitabine in combination with oxaliplatin and pelvic radiation (XELOX-RT) in patients with locally advanced rectal cancer. Ann Oncol 17: $50-56$

Lissoni P, Meregalli S, Bonetto E, Mancuso M, Brivio F, Colciago M, Gardani G (2005) Radiotherapy-induced lymphocytopenia: changes in total lymphocyte count and in lymphocyte subpopulations under pelvic irradiation in gynecologic neoplasms. J Biol Regul Homeost Agents 19: $153-158$

Machiels JP, Duck L, Honhon B, Coster B, Coche JC, Scalliet P, Humblet Y, Aydin S, Kerger J, Remouchamps V, Canon JL, Van Maele P, Gilbeau L, Laurent S, Kirkove C, Octave-Prignot M, Baurain JF, Kartheuser A,
Sempoux C (2005) Phase II study of preoperative oxaliplatin, capecitabine and external beam radiotherapy in patients with rectal cancer: the RadiOxCape study. Ann Oncol 16: 1898-1905

Mandard AN, Dalibard F, Mandard JC, Marnay J, Henry-Amar M, Petiot JF, Roussel A, Jacob JH, Segol P, Samama G (1994) Pathological assessment of tumour regression after preoperative chemoradiotherapy of esophageal carcinoma: clinico-pathologic correlations. Cancer 73: $2680-2686$

Roedel C, Grabenbauer GG, Papadopoulos T, Hohenberger W, Schmoll HJ, Sauer R (2003) Phase I/II trial of capecitabine, oxaliplatin and radiation for rectal cancer. J Clin Oncol 21: 3098-3104

Roedel C, Liersch T, Hermann RM, Arnold D, Reese T, Hipp M, Fürst A, Schwella N, Bieker M, Hellmich G, Ewald H, Haier J, Lordick F, Flentje M, Sülberg H, Hohenberger W, Sauer R (2007) Multicenter phase II trial of chemoradiation with oxaliplatin for rectal cancer. J Clin Oncol 25: $110-117$

Roedel C, Martus P, Papadoupolos T, Füzesi L, Klimpfinger M, Fietkau R, Liersch T, Hohenberger W, Raab R, Sauer R, Wittekind C (2005) Prognostic significance of tumor regression after preoperative chemoradiotherapy for rectal cancer. J Clin Oncol 23: 8688-8696

Roh MS, Colangelo L, Wieand S, O'Connell M, Petrelli N, Smith R, Mamounas E, Hyams D, Wolmark N (2004) Response to preoperative multimodality therapy predicts survival in patients with carcinoma of the rectum. J Clin Oncol 22(14S): (abstract 3505)

Rutten H, Sebag-Montefiore D, Glynne-Jones R, Rullier E, Peeters M, Brown G, Van Cutsem E, Ricci S, Van de Velde CJ, Quirke P (2006) Capecitabine, oxaliplatin, radiotherapy, and excision (CORE) in patients with MRI-defined locally advanced rectal adenocarcinoma: results of an international multicenter phase II study. J Clin Oncol 24(18S): (abstract 3528)

Sauer R, Becker H, Hohenberger W, Rödel C, Wittekind C, Fietkau R, Martus P, Tschmelitsch J, Hager E, Hess CF, Karstens JH, Liersch T, Schmidberger H, Raab R, German Rectal Cancer Study Group (2004) Preoperative $v s$ postoperative chemoradiotherapy for rectal cancer. $N$ Engl J Med 351: $1731-1740$

Wheeler JM, Dodds E, Warren BF, Cunningham C, George BD, Jones AC, Mortensen NJ (2004) Preoperative chemoradiotherapy and total mesorectal excision surgery for locally advanced rectal cancer: correlation with rectal cancer regression grade. Dis Colon Rectum 47: $2025-2031$ 